PROCEEDINGS OF THE

AMERICAN MATHEMATICAL SOCIETY

Volume 125, Number 8, August 1997, Pages 2285-2291

S 0002-9939(97)03763-5

\title{
GROTHENDIECK OPERATORS ON TENSOR PRODUCTS
}

\author{
P. DOMAŃSKI, M. LINDSTRÖM, AND G. SCHLÜCHTERMANN
}

(Communicated by Palle E. T. Jorgensen)

\begin{abstract}
We prove that for Banach spaces $E, F, G, H$ and operators $T \in$ $\mathcal{L}(E, G), S \in \mathcal{L}(F, H)$ the tensor product $T \otimes S: E \otimes_{\varepsilon} F \rightarrow G \otimes_{\varepsilon} H$ is a Grothendieck operator, provided $T$ is a Grothendieck operator and $S$ is compact.
\end{abstract}

\section{INTRODUCTION}

J. Diestel and B. Faires proved in '76 that for Banach spaces $E, F, G, H$, for $T \in \mathcal{A}(E, G)$ and compact $S \in \mathcal{L}(F, H)$ the tensor product of $T$ and $S$ defined as $T \otimes S: E \otimes_{\varepsilon} F \rightarrow G \otimes_{\varepsilon} H$ belongs again to the operator ideal $\mathcal{A}$, provided $\mathcal{A}$ is closed and injective $[\mathrm{DF}]$. For the ideal of weakly compact operators E. Saksman and H. O. Tylli $[\mathrm{ST}]$ have obtained similar results for both the projective and injective tensor product.

The mentioned results open a natural, and interesting in itself, question on stability of non-injective operator ideals with respect to injective tensor products. We solve this problem for the non-injective, closed ideal of Grothendieck operators. We are interested in exactly that ideal because the corresponding problem of tensor stability turns out to be closely related to the question of existence of complemented copies of $c_{0}$ in injective tensor products even for Fréchet spaces. In fact, it was shown [R, p. 98] that for a large class of Banach spaces $E$ (containing all $E=C(K)$ ) we have that $E$ is a Grothendieck space (that is, weak* and weak sequential convergence coincide on equicontinuous subsets) if and only if $E$ contains no complemented copy of $c_{0}$.

On the other hand, by a surprising result of Freniche [Fr1] (compare [C]), each completed injective tensor product $E \otimes_{\varepsilon} F$ of a Fréchet space $E$ containing a copy of $c_{0}$ and a Fréchet space $F$ satisfying the Josefson-Nissenzweig type theorem (that is, weak* and strong convergence do not coincide for sequences in the dual) contains always a complemented copy of $c_{0}$. A fortiori, such a tensor product cannot be a Grothendieck space. All infinite dimensional Banach spaces satisfy the JosefsonNissenzweig theorem and for Fréchet spaces it was proved by Bonet, Lindström and Valdivia $[\mathrm{BLV}]$ that this property exactly characterizes the non-Montel spaces.

This development leads to two natural questions: Let $E$ be a Fréchet space and $F$ a Fréchet-Montel space. When exactly does $E \otimes_{\varepsilon} F$ contain a complemented copy of $c_{0}$ and when exactly is it a Grothendieck space? Both problems can also be interpreted in terms of tensor stability.

Received by the editors August 29, 1995 and, in revised form, January 9, 1996.

1991 Mathematics Subject Classification. Primary 47A80.

(C)1997 American Mathematical Society 
In case of $E=C(K)$ it follows immediately from results of Freniche [Fr2] (compare [DL, Cor. 3.7]) that $C(K, F)$ is a Grothendieck space if and only if $C(K)$ is a Grothendieck space which automatically implies that $C(K, F)$ contains a complemented copy of $c_{0}$ if and only if $C(K)$ contains a complemented copy of $c_{0}$.

For general injective tensor products, the known results are contained in [DL, Th. 2.3, Th. 3.6]:

(i) if $F$ has the approximation property, then $E \otimes_{\varepsilon} F$ contains a complemented copy of $c_{0}$ if and only if $E$ contains a complemented copy of $c_{0}$.

(ii) if either $F$ or $E^{\prime \prime}$ has the approximation property, then $E \otimes_{\varepsilon} F$ is a Grothendieck space if and only if $E$ is a Grothendieck space.

Using our injective tensor stability result for the ideal of Grothendieck operators we are able to remove the approximation property assumption from the second result when $E$ is a Banach space and $F$ is a Schwartz space.

Let us now fix some notations and definitions. $E, F, G, H$ are Banach spaces. $B(E)$ stands for the unit ball, while $E^{*}$ denotes the topological dual. By an operator $T$ from $E$ into $F$ we mean a bounded linear map. Let us call an operator $T \in$ $\mathcal{L}(E, F)$ approximable if there exists a sequence of finite rank operators $\left(v_{n}\right) \subset$ $\mathcal{L}(E, F)$ such that $\left\|T-v_{n}\right\|^{n \rightarrow \infty} \rightarrow 0$ (cf. [Jh]). We refer to [Pi], [DU] and [DFl] for background information on operator ideals, measure theory and tensor products, respectively.

Definition 1.1. Let $E, F$ be Banach spaces. An operator $T \in \mathcal{L}(E, F)$ is called a Grothendieck operator if every $w^{*}$-null sequence $\left(y_{n}^{*}\right)$ is mapped by the adjoint $T^{*}$ into a weak null sequence $\left(T^{*}\left(y_{n}^{*}\right)\right) \subset E^{*}$.

The ideal of Grothendieck operators $\mathcal{G R}(E, F)$ is not injective, since the inclusion map $\iota: c_{0} \hookrightarrow \ell_{\infty}$ is Grothendieck (note that $\ell_{\infty}$ is a Grothendieck space, since $w^{*}$ null sequences are weakly null in the dual of $\ell_{\infty}$ ). But the identity $i d: c_{0} \rightarrow c_{0}$ is not Grothendieck, since otherwise it would be weakly compact.

Definition 1.2. A subset $K \subset E$ is called a Grothendieck set if for all $T \in \mathcal{L}\left(E, c_{0}\right)$ the set $T(K)$ is relatively weakly compact in $c_{0}$.

We have immediately the following result (cf. [DU, p. 179]).

Lemma 1.3. Let $E, F$ be Banach spaces, $T \in \mathcal{L}(E, F)$. Then the following conditions are equivalent:

(a) $T \in \mathcal{G} \mathcal{R}(E, F)$,

(b) $\forall S \in \mathcal{L}\left(F, c_{0}\right): S \circ T$ is weakly compact,

(c) $\forall A \subset E$ bounded: $T(A)$ is a Grothendieck set.

It follows that the ideal $\mathcal{G R}$ is surjective and closed.

\section{MAIN RESUlts}

Let $\mathcal{A}$ be a closed operator ideal and $\alpha$ be a tensor norm. We define the class $\mathcal{A}_{\alpha}$ of all operators $S: E \rightarrow F$ such that for any pair of Banach spaces $E_{1}, F_{1}$ and any operator $T \in \mathcal{A}\left(E_{1}, F_{1}\right)$ the map $T \otimes S: E_{1} \otimes_{\alpha} E \rightarrow F_{1} \otimes_{\alpha} F$ belongs to $\mathcal{A}$ as well. J. Diestel and B. Faires (see [DF, Th. 1 and Th. 2]) proved that $\mathcal{A}_{\alpha}$ is always a closed operator ideal which is injective whenever $\mathcal{A}$ and $\alpha$ are injective. Analogously, it is easily seen that if $\mathcal{A}$ is surjective and $\alpha$ is projective, then $\mathcal{A}_{\alpha}$ is surjective. Thus we obtain immediately: 
Proposition 2.1. Let $E, F, G, H$ be Banach spaces, $\mathcal{A}$ be a closed operator ideal, $\alpha$ be a tensor norm and $T \in \mathcal{A}(E, G)$.

(a) If $S \in \mathcal{L}(F, H)$ is approximable, then $T \otimes S: E \otimes_{\alpha} F \rightarrow G \otimes_{\alpha} H$ is again in $\mathcal{A}$.

(b) If $\alpha$ and $\mathcal{A}$ are injective, then (a) holds even for compact $S$.

(c) If $\alpha$ is projective and $\mathcal{A}$ is surjective, then (a) holds even for compact $S$.

Proof. The ideal of approximable operators is the smallest closed operator ideal. Similarly, the ideal of compact operators is the smallest surjective (injective) closed operator ideal.

Since $\mathcal{G} \mathcal{R}$ is surjective, we can state the following result.

Corollary 2.2. Let $E, F, G, H$ be Banach spaces. Then $T \otimes S: E \otimes_{\pi} F \rightarrow G \otimes_{\pi} H$ belongs to $\mathcal{G} \mathcal{R}$ whenever $T$ is Grothendieck and $S$ is compact.

Remark 2.3. If we apply Theorem 2.2 and Remark 2.9 of $[\mathrm{S}]$ the following criterion of weak compactness in the dual space $\left(E \otimes_{\pi} F\right)^{*}=\mathcal{L}\left(E, F^{*}\right)$ can be obtained: Let $\left(T_{n}\right) \subset \mathcal{L}\left(E, F^{*}\right)$ be a bounded sequence. Then $T_{n} \stackrel{n \rightarrow \infty}{\rightarrow} 0$ weakly if and only if $\left\{\left(\left\langle T_{n}(x), y^{* *}\right\rangle\right)_{n \in \mathbb{N}} ; x \in B(E), y^{* *} \in B\left(F^{* *}\right)\right\} \subset c_{0}$ is relatively weakly compact. This result or Theorem 1 in $[\mathrm{K}]$ can be used to give a direct proof of the above corollary. It also follows from the proof that $E \otimes_{\pi} F$ is a Grothendieck space if $E$ is a Grothendieck space, $F$ is reflexive and every operator from $E$ into $F^{*}$ is compact.

At this stage we mention that from P. Enflo's famous example [E] it is an easy consequence that there is a Banach space $E$ for which there is a non-approximable but compact operator from $E$ into itself. In $[\mathrm{A}] \mathrm{F}$. A. Alexander obtained a similar result for a closed subspace $E$ of $l^{p}$ when $2<p<\infty$.

The ideal of Grothendieck operators is not injective. Thus our main aim is to improve 2.1 in that case and to obtain injective tensor stability with compact operators. First we reduce the problem to reflexive $F$ and $H$.

Lemma 2.4. Let $E, F, G, H$ be Banach spaces, $T \in \mathcal{L}(E, G)$ and $S \in \mathcal{L}(F, H)$ is compact. Then there exist reflexive Banach spaces $G_{1}, H_{1}$ and operators $S_{1} \in$ $\mathcal{L}\left(E, G_{1}\right), S_{2} \in \mathcal{L}\left(G_{1}, H_{1}\right), S_{2}$ compact, $S_{3} \in \mathcal{L}\left(H_{1}, H\right)$, such that

$$
T \otimes S=\left(i d_{G} \otimes S_{3}\right) \circ\left(T \otimes S_{2}\right) \circ\left(i d_{E} \otimes S_{1}\right) .
$$

Proof. Every compact $S \in \mathcal{L}(F, H)$ admits a compact factorization through a reflexive Banach space according to a result of T. Figel and W. Johnson [Fi, Jo] (see also [DU, p. 260]). Then the proof is straightforward.

We write $B o\left(B\left(E^{*}\right)\right)$ for the Borel sets on $B\left(E^{*}\right)$ w.r.t. the $w^{*}$-topology. If $m: B o\left(B\left(X^{*}\right)\right) \rightarrow F$ is a vector measure of bounded variation, then $\|m\|$ is the variation norm. Let us recall the representation of the dual of $E \otimes_{\varepsilon} F$, provided $F$ is reflexive.

Definition and Lemma 2.5. Let $E, F$ be Banach spaces with $F$ reflexive. $\mathcal{P} \mathcal{I}(E, F) \subset \mathcal{L}(E, F)$ are the Pietsch-integral operators, defined as:

$$
\begin{gathered}
T \in \mathcal{P} \mathcal{I}(E, F) \Leftrightarrow \exists m: B o\left(B\left(E^{*}\right)\right) \rightarrow F \text { vector measure of bounded variation } \\
\forall x \in E: T(x)=\int_{B\left(E^{*}\right)} x\left(x^{*}\right) d m\left(x^{*}\right) .
\end{gathered}
$$


We equip $\mathcal{P} \mathcal{I}(E, F)$ with the integral norm, i.e. $\|T\|_{P I}:=\inf \{\|m\| ; \forall x \in E: T(x)=$ $\left.\int_{B\left(E^{*}\right)} x\left(x^{*}\right) d m\left(x^{*}\right)\right\}$ (cf. [DFl, p. 522]). Then $\mathcal{P} \mathcal{I}\left(E, F^{*}\right)$ is isometric isomorphic to $\left(E \otimes_{\varepsilon} F\right)^{*}$ by the identity $T(x \otimes y)=\left\langle y, \int_{B\left(E^{*}\right)} x\left(x^{*}\right) d m\left(x^{*}\right)\right\rangle(c f$. [DFl, p. 522]).

Notation. Let $E, F$ be Banach spaces with $F$ reflexive, and let $\left(z_{n}^{*}\right) \subset$ $B\left(\left(E \otimes_{\varepsilon} F\right)^{*}\right)$. According to 2.5 for all $n \in \mathbb{N}$ we choose a vector measure $m_{n}:=$ $m_{n}\left(z_{n}^{*}\right): B o\left(B\left(X^{*}\right)\right) \rightarrow F$ of bounded variation, satisfying:

i) $\lim _{n \rightarrow \infty}\left|\left\|m_{n}\right\|-\left\|z_{n}^{*}\right\|\right|=0$,

ii) $\forall e \in E, f \in F, n \in \mathbb{N}: z_{n}^{*}(e \otimes f)=\left\langle f, \int_{B\left(E^{*}\right)} e\left(e^{*}\right) d m_{n}\left(e^{*}\right)\right\rangle$.

Furthermore we define a finite scalar-valued measure $\mu(\cdot):=\mu\left(\left(z_{n}^{*}\right)\right)(\cdot):=$ $\sum_{n \in \mathbb{N}} 2^{-n} \operatorname{var}\left(m_{n}\left(z_{n}^{*}\right), \cdot\right)$, where var denotes the variation of the corresponding measure. Then $m_{n}$ is absolutely continuous w.r.t. $\mu$ for all $n \in \mathbb{N}$.

We write

$$
L_{H_{1}}:=\left\{f \in L_{1}\left(\mu, H_{1}^{*}\right): \forall e \in E: \int_{B\left(E^{*}\right)} e\left(e^{*}\right) f\left(e^{*}\right) d \mu\left(e^{*}\right)=0\right\}
$$

for a subspace $H_{1} \subset H$. For a Banach space $H$ we denote by $q_{H}: L_{1}\left(\mu, H^{*}\right) \rightarrow$ $L_{1}\left(\mu, H^{*}\right) / L_{H}$ the canonical quotient map. If $\mu=\mu\left(z_{n}^{*}\right)$ and $H_{1} \subset H$, then let $r_{H_{1}}: L_{1}\left(\mu, H_{1}^{*}\right) / L_{H_{1}} \rightarrow\left(E \otimes_{\varepsilon} H_{1}\right)^{*}$ be the canonical injection.

Theorem 2.6. Let $E, F, G, H$ be Banach spaces. If $T \in \mathcal{G R}(E, G)$ and $S \in$ $\mathcal{L}(F, H)$ is compact, then $T \otimes S: E \otimes_{\varepsilon} F \rightarrow G \otimes_{\varepsilon} H$ is Grothendieck.

Proof. By Lemma 2.4 we assume that $F, H$ are reflexive. W.l.o.g. let $\|T\|,\|S\| \leq 1$. Let $\left(x_{n}^{*}\right) \subset B\left(\left(G \otimes_{\varepsilon} H\right)^{*}\right)$ be $w^{*}$-converging to 0 . First we consider the map $T \otimes i d: E \otimes_{\varepsilon} H \rightarrow G \otimes_{\varepsilon} H$. For a finite dimensional subspace $H_{1} \subset H$, according to 2.1 , we have that for $\left(z_{n}^{*}\right):=(T \otimes i d)^{*}\left(x_{n}^{*}\right)$ the restriction

$$
\left(\left.\left(z_{n}^{*}\right)\right|_{E \otimes H_{1}}\right) \stackrel{n \rightarrow \infty}{\longrightarrow} 0 \text { weakly. }
$$

Consider now $i d \otimes S: E \otimes_{\varepsilon} F \rightarrow E \otimes_{\varepsilon} H$. For $n \in \mathbb{N}$ let $h_{n} \in L_{1}\left(\mu, H^{*}\right)$ be the density of $m_{n}$ with respect to $\mu:=\mu\left(z_{n}^{*}\right)$. To show that $(i d \otimes S)^{*}\left(\left(z_{n}^{*}\right)\right)$ is weakly null (then we are done), we have to show that for all $\bar{g} \in B\left(\left(E \otimes_{\varepsilon} F\right)^{* *}\right)$ :

$$
\begin{aligned}
\left\langle\bar{g}(i d \otimes S)^{*}\left(z_{n}^{*}\right)\right\rangle & =\int_{B\left(E^{*}\right)}\left\langle q_{F}^{*} \circ r_{F}^{*}(\bar{g}), S^{*} \circ h_{n}\right\rangle d \mu \\
& =\int_{B\left(E^{*}\right)}\left\langle S \circ q_{F}^{*} \circ r_{F}^{*}(\bar{g}), h_{n}\right\rangle d \mu \rightarrow 0 .
\end{aligned}
$$

The following arguments are devoted to proving this. We define $g:=S \circ q_{F}^{*} \circ$ $r_{F}^{*}(\bar{g})$. Then $g \in L_{\infty}(\mu, H)$, since $H$ is reflexive. Further, $g$ has relatively compact range, since $S$ is compact. We assume that (2) is not true. Then

$$
\exists\left(h_{n_{k}}\right) \text { subsequence } \exists \varepsilon>0: \inf _{k \in \mathbb{N}}\left|\int_{B\left(E^{*}\right)}\left\langle g, h_{n_{k}}\right\rangle d \mu\right|>\varepsilon .
$$

For the sake of simplicity assume that $\left(h_{n}\right)$ satisfies (3). Since $g$ has relatively compact range, there is an increasing sequence of finite $B o\left(B\left(E^{*}\right)\right)$-partitions $\left(\pi_{k}\right)$, such that

$$
\left\|\mathbb{E}_{\pi_{k}}(g)-g\right\|_{\infty} \rightarrow 0 \text { and } \forall n \in \mathbb{N}:\left\|\mathbb{E}_{\pi_{k}}\left(h_{n}\right)-h_{n}\right\|_{1} \rightarrow 0
$$


We define $\Sigma_{0}:=\sigma\left(\bigcup_{k \in \mathbb{N}} \pi_{k}\right)$. Since $H$ is reflexive, for all $k \in \mathbb{N}$ the sequence $\left(\mathbb{E}_{\pi_{k}}\left(h_{n}\right)\right)$ is relatively weakly compact in $L_{1}\left(\mu, H^{*}\right)$. Hence, for all $k \in \mathbb{N}$ there is an $m_{k} \in L_{1}\left(\mu, H^{*}\right)$, so that $\mathbb{E}_{\pi_{k}}\left(h_{n}\right) \rightarrow m_{k}$ weakly (for at least going to a subsequence by a diagonalization argument). $\left(\pi_{k}\right)$ is increasing, thus, $\left(m_{k}, \pi_{k}\right)$ is a bounded martingale, which converges in the $L_{1}\left(\mu, H^{*}\right)$-norm to an $M \in L_{1}\left(\mu, H^{*}\right)$ (note that the $\left(h_{n}\right)$ are bounded and $H^{*}$ has the RNP as a reflexive space). We show now that for all $G \in L_{\infty}\left(\left.\mu\right|_{\sum_{0}}, H\right)$ with relatively compact range:

$$
\begin{aligned}
& \exists \text { subsequence }\left(h_{n_{j}}\right) \text { such that } \\
& \quad \forall \delta>0 \exists N \in \mathbb{N} \forall j \geq N:\left|\langle G, M\rangle-\left\langle G, h_{n_{j}}\right\rangle\right|<\delta .
\end{aligned}
$$

Proof of (5). $G$ has relatively compact range, thus there exists an increasing sequence of finite $B o\left(B\left(E^{*}\right), w^{*}\right)$-partitions $\left(\pi_{k}(G)\right)$ such that $\pi_{k} \subset \sigma\left(\pi_{k}(G)\right)$ for $k \in$ $\mathbb{N}$ and $\left\|G-\mathbb{E}_{\pi_{k}(G)}(G)\right\| \rightarrow 0$ (cf. [DU, p. 67 , Lemma 1]). Let $\left(h_{n_{j}}\right)$ be a subsequence with $\mathbb{E}_{\pi_{k}(G)}\left(h_{n_{j}}\right) \rightarrow m_{k}(G) \in L_{1}\left(\mu, H^{*}\right)$ weakly for all $k \in \mathbb{N}$ (subsequence argument like above). Thus, since $\pi_{k} \subset \sigma\left(\pi_{k}(G)\right)$, it follows $\mathbb{E}_{\pi_{k}}\left(\mathbb{E}_{\pi_{k}(G)}\left(h_{n_{j}}\right)\right) \rightarrow m_{k}$ $\left(m_{k}\right.$ as above). Again $\left(m_{k}(G)\right)$ is a martingale. Hence, as above, there exists an $M(G) \in L_{1}\left(\mu, H^{*}\right)$, such that $m_{k}(G) \rightarrow M(G)$. We have

$$
M=M(G) \text {. }
$$

Let $A \in \bigcup_{k \in \mathbb{N}} \pi_{k}$. Then

$$
\int_{A} M(G) d \mu-\int_{A} M d \mu=\lim _{k \rightarrow \infty} \int_{A} m_{k}(G)-m_{k} d \mu=0,
$$

since $\left(m_{k}(G)\right)$ and $\left(m_{k}\right)$ are martingales and there is a $k_{0} \in \mathbb{N}$, such that $A \in \pi_{k_{0}} \subset$ $\sigma\left(\pi_{k}(G)\right)$. Hence, for all $B \in \Sigma_{0}: \int_{B} M(G) d \mu=\int_{B} M d \mu$. Thus to prove (5) we first note that it suffices to demonstrate (5) for all $G=\mathbb{E}_{\pi_{k}(G)} G(k \in \mathbb{N})$, since $G$ has relatively compact range and $M, h_{n}, n \in \mathbb{N}$, are measurable w.r.t. $\Sigma_{0}$. But then (5) follows by:

$$
\begin{aligned}
\left\langle\mathbb{E}_{\pi_{k}(G)} G, M\right\rangle-\left\langle\mathbb{E}_{\pi_{k}(G)} G, h_{n_{j}}\right\rangle & =\left\langle\mathbb{E}_{\pi_{k}(G)} G, m_{k}(G)\right\rangle-\left\langle\mathbb{E}_{\pi_{k}(G)} G, \mathbb{E}_{\pi_{k}(G)} h_{n_{j}}\right\rangle \\
& =\left\langle\mathbb{E}_{\pi_{k}(G)} G, m_{k}(G)-\mathbb{E}_{\pi_{k}(G)} h_{n_{j}}\right\rangle .
\end{aligned}
$$

For a finite dimensional subspace $H_{1} \subset H$ we consider the canonical restriction operator $\operatorname{rest}_{H_{1}}: L_{1}\left(\mu, H^{*}\right) / L_{H^{*}} \rightarrow L_{1}\left(\mu, H_{1}^{*}\right) / L_{H_{1}^{*}}$. Then according to (1) we have:

$$
\forall z^{* *} \in\left(E \otimes_{\varepsilon} H_{1}\right)^{* *}: \int_{B\left(E^{*}\right)}\left\langle q_{H}^{*} \circ \operatorname{rest}_{H_{1}}^{*} \circ r_{H_{1}}^{*}\left(z^{* *}\right), h_{n}\right\rangle d \mu \rightarrow 0 .
$$

Since $q_{H}^{*} \circ$ rest $H_{1} \circ r_{H_{1}}^{*}\left(z^{* *}\right)$ has relatively compact range for all $z^{* *} \in\left(E \otimes_{\varepsilon} H_{1}\right)^{* *}$ ( $H_{1}$ is finite dimensional), (5) and (6) imply:

$$
\forall z^{* *} \in\left(E \otimes_{\varepsilon} H_{1}\right)^{* *}:\left\langle z^{* *}, r_{H_{1}} \circ \operatorname{rest}_{H_{1}} \circ q_{H}(M)\right\rangle=0 .
$$

Note that since $M \in L_{1}\left(\left.\mu\right|_{\Sigma_{0}}, H^{*}\right)$ we may assume that $q_{H}^{*} \circ$ rest $_{H_{1}}^{*} \circ r_{H_{1}}^{*}\left(z^{* *}\right)$ is measurable w.r.t. $\Sigma_{0}$. Thus

$$
\forall H_{1} \subset H \text { finite dimensional } r_{H_{1}} \circ \operatorname{rest}_{H_{1}} \circ q_{H}(M)=0 .
$$

But (7) implies

$$
r_{H} \circ q_{H}(M)=0 .
$$


Hence we compute

$$
\begin{aligned}
0 & \stackrel{(8)}{=}\left\langle(i d \otimes S)^{* *}(\bar{g}), r_{H} \circ q_{H}(M)\right\rangle=\left\langle\bar{g},(i d \otimes S)^{*}\left(r_{H} \circ q_{H}(M)\right)\right\rangle \\
& =\int_{B\left(E^{*}\right)}\left\langle q_{F}^{*} \circ r_{F}^{*}(\bar{g}), S^{*} \circ M\right\rangle d \mu=\int_{B\left(E^{*}\right)}\left\langle S \circ q_{F}^{*} \circ r_{F}^{*}(\bar{g}), M\right\rangle d \mu \\
& =\int_{B\left(E^{*}\right)}\langle g, M\rangle d \mu .
\end{aligned}
$$

Thus, this contradicts (3) and (5), and we are done.

We shall now apply Theorem 2.6 and an operator ideal approach to obtain the announced result avoiding the assumption of the approximation property.

Corollary 2.7. Let $E$ be a Schwartz space and $F$ a Banach space with the Grothendieck property. Then $E \otimes_{\varepsilon} F$ is a Grothendieck space.

Proof. By a well-known representation of $\varepsilon$-tensor products as projective limits $E \otimes_{\varepsilon} F=\operatorname{proj}_{U \in \mathcal{U}_{E}} E_{U} \otimes_{\varepsilon} F$, where $\mathcal{U}_{E}$ is a 0 -basis in $E$. A locally convex space $X$ is Grothendieck if and only if every continuous linear map from $X$ into $c_{0}$ maps bounded sets into relatively weakly compact ones. Now, each continuous linear map $T: E \otimes_{\varepsilon} F \rightarrow c_{0}$ factorizes through $E_{U} \otimes_{\varepsilon} F$ for some $U \in \mathcal{U}_{E}$. Since $E$ is a Schwartz space we can apply our main theorem so that for every $U \in \mathcal{U}_{E}$ there exists a $V \in \mathcal{U}_{E}$ contained in $U$ such that the canonical map $E_{V} \otimes_{\varepsilon} F \rightarrow E_{U} \otimes_{\varepsilon} F$ is a Grothendieck operator. The result follows immediately.

\section{REFERENCES}

[A] F. A. Alexander, Compact and finite rank operators on subspaces of $l^{p}$, Bull. London Math. Soc. 6 (1974), 341-342. MR 50:8135

[BLV] J. Bonet, M. Lindström, and M. Valdivia, Two theorems of Josefson-Nissenzweig type for Fréchet spaces, Proc. Amer. Math. Soc. 117 (1993), 363-364. MR 93d:46005

[C] P. Cembranos, $C(K, E)$ contains a complemented copy of $c_{0}$, Proc. Amer. Math. Soc. 91 (1984), 556-558. MR 85g:46025

[DL] P. Domański and M. Lindström, Grothendieck spaces and duals of injective tensor products, Bull. London Math. Soc. 28 (1996), 617-626. CMP 96:17

[DF] J. Diestel and B. Faires, Remarks on the classical Banach operator ideals, Proc. Amer. Math. Soc. 58 (1976), 189-196. MR 56:12950

[DFl] A. Defant and K. Floret, Tensor Norms and Operator Ideals, North-Holland (1993). MR 94e: 46130

[DU] J. Diestel and J. Uhl, Vector measures, AMS Math. Surveys 15 (1977). MR 56:12216

[E] P. Enflo, A counterexample to the approximation problem in Banach spaces, Acta Math. 130 (1973), 309-317. MR 53:6288

[Fi] T. Figiel, Factorization of compact operators and applications to the approximation problem, Studia Math. 45 (1973), 191-210. MR 49:1070

[Fr1] F. J. Freniche, Barrelledness of the spaces of vector-valued and simple functions, Math. Ann. 267 (1984), 479-486. MR 85f:46074

[Fr2] F. J. Freniche, Grothendieck locally convex spaces of continuous vector-valued functions, Pacific J. Math. 120 (1985), 345-355. MR 87a:46059

[Jh] K. John, On the compact non-nuclear operator problem, Math. Ann. 287 (1990), 509-514. MR 91f: 47029

[Jo] W. Johnson, Factoring compact operators, Isr. J. Math. 9 (1971), 337-345. MR 44:7318

[K] N. Kalton, Spaces of compact operators, Math. Ann. 208 (1974), 267-278. MR 49:5904

[Pi] A. Pietsch, Operator ideals, North-Holland (1980). MR 81j:47001

[R] F. Räbiger, Beiträge zur Strukturtheorie der Grothendieckräume, Sitzungsber. Heidelb. Akad. Wiss. Math.-Natur. Kl. Abl. 4 (1985). MR 87g:46035 
[ST] E. Saksman and H. O. Tylli, Weak compactness of multiplication operators on spaces of bounded linear operators, Math. Scand. 70 (1992), 91-111. MR 93j:46080

[S] G. Schlüchtermann, Weak compactness in $L_{\infty}(\mu, X)$, J. Funct. Anal. 125 (1994), 379-388. MR 95f: 46055

Department of Mathematics, A. Mickiewicz University, 60-769 Poznań, Poland

E-mail address: domanski@math.amu.edu.pl

Department of Mathematics, Åbo Akademi University, Fin-20500 Åbo, Finland

E-mail address: mikael.lindstrom@abo.fi

Mathematisches Institut Der Universität München, Theresienstrasse 39 , D-80333 MüNChen, GeRMANY

E-mail address: schluech@rz.mathematik.uni-muenchen.de 